The Astrophysical Journal, 248:1179-1193, 1981 September 15

(C) 1981. The American Astronomical Society. All rights reserved. Printed in U.S.A.

\title{
A MEASUREMENT OF THE COSMIC-RAY ANTIPROTON FLUX AND A SEARCH FOR ANTIHELIUM
}

\author{
Andrew Buffington, ${ }^{1,2}$ Stephen M. Schindler, ${ }^{1}$ And Carlton R. Pennypacker ${ }^{2}$ \\ Received 1981 February 4; accepted 1981 March 27
}

\begin{abstract}
A balloon-borne instrument has measured the cosmic-ray antiproton flux between 130 and 320 $\mathrm{MeV}$ and searched for antihelium between 130 and $370 \mathrm{MeV}$ per nuclear. These particles were selected from the background of normal-matter cosmic rays by combining a selective trigger with a detailed spark chamber visualization of each recorded event. Antiprotons are identified by their characteristic annihilation radiation. Residue from background processes meeting the selection criteria is small. The observed 14 antiprotons yield a measured differential flux of $1.7 \pm 0.5 \times 10^{-4}$ antiprotons $\mathrm{m}^{-2} \mathrm{sr}^{-1} \mathrm{~s}^{-1} \mathrm{MeV}^{-1}$ at the top of the atmosphere. The corresponding antiproton/proton ratio is $2.2 \pm 0.6 \times 10^{-4}$, only slightly smaller than the ratio observed by other experiments at higher energies. Thus the antiprotons have a spectral shape similar to the protons, at least down to about $100 \mathrm{MeV}$. The expected flux of these particles can be calculated under the assumption that they were created by collisions of high-energy cosmic rays with the interstellar gas. Calculations using the standard leaky box model for propagation in the Galaxy predict a flux two orders of magnitude smaller than that observed. A small low-energy flux is predicted due to a kinematic suppression of the production of low-energy antiprotons. The discrepancy between calculations and experiment may be evidence that cosmic-ray protons have passed through substantially more than $5 \mathrm{~g} \mathrm{~cm}^{-2}$ of material during their lifetime. In addition, the combined results from this experiment and previous ones may be evidence for stochastic, energy-changing processes in interstellar space which act upon the secondary antiprotons after their creation. The search for cosmic-ray antihelium sets a 95\% confidence level upper limit on the $\overline{\mathrm{He}} / \mathrm{He}$ ratio of $2.2 \times 10^{-5}$.
\end{abstract}

Subject headings: cosmic rays: abundances - elementary particles - particle acceleration

\section{INTRODUCTION}

The symmetry between matter and antimatter observed in accelerator experiments has stimulated a great interest in its possible cosmological consequences. Steigman (1976) has reviewed a number of models dealing with creation and subsequent evolution of this "cosmological antimatter" and has discussed the observations that could indicate whether such antimatter exists. He concludes that there is at present no observational evidence in favor of cosmological antimatter. Furthermore, Steigman shows that the observed $\gamma$-ray fluxes set stringent limits on the amount of mixing allowed between matter and antimatter regions.

The search for cosmological antimatter is difficult because the symmetry between matter and antimatter makes regions composed of each indistinguishable when observed through the normal photon channels. As a result, there has been major interest in searching among the high-energy cosmic rays for antiparticles. Most of the observed galactic cosmic rays are believed to have

\footnotetext{
${ }^{1}$ California Institute of Technology

${ }^{2}$ University of California, Berkeley.
}

come from within about $10^{3} \mathrm{pc}$ of the solar system, although a portion may be extragalactic (see, for example, Ginzburg and Syrovatskii 1964, chap. 3). Thus, the cosmic rays can be-employed as a probe of the matter/antimatter composition of a region extending far beyond our solar system. Already, balloon-borne magnetic spectrometers have examined about $10^{5}$ highenergy cosmic-ray nuclei (with charge $Z \geq 2$ ) and have observed no antiparticles (Smoot, Buffington, and Orth 1975; Badhwar et al. 1978). Even so, the importance of cosmological antimatter provides a continuing motivation for experiments that can extend the search for antinuclei in the cosmic rays. This is, however, only the secondary objective of the experiment described in this paper.

The primary objective here is the measurement of antimatter that is expected to occur in the cosmic rays independent of cosmological effects. High-energy cosmic rays, mostly protons, interact with interstellar gas, creating a small flux of positrons and antiprotons $(\bar{P})$ by the same process that led to the discovery of antimatter in accelerator experiments. Positrons have been observed at about the expected fluxes (Fanselow et al. 1969; Buffington, Orth, and Smoot 1975). A number of calcu- 
lations predict a cosmic-ray antiproton to proton $(\bar{P} / P)$ ratio of about $2 \times 10^{-4}$ above several GeV, typically a tenth of the $e^{+} / P$ ratio (Gaisser and Maurer 1973; Gaisser and Levy 1974; Badhwar et al. 1975; Szabelski, Wdowczyk, and Wolfendale 1980; Stephens 1980). Golden et al. (1979) recently reported a finite flux of antiprotons at approximately this expected level. Bogomolov et al. (1979) also have reported an observation of cosmic-ray antiprotons, but that measurement was based on only two events.

Antiproton measurements are important because these particles are mostly daughters of interactions of cosmicray protons. On the other hand, most of our knowledge of cosmic-ray history comes from studies of heavier secondary nuclei such as $\mathrm{Li}, \mathrm{Be}, \mathrm{B}$, and the sub-iron elements, whose heavy-nucleus parents have not necessarily had the same history as the much more plentiful protons. Positrons could serve as tracers of proton cosmic-ray history, since their parents (as with the antiprotons) are mostly protons. However, the positron spectrum is distorted by electromagnetic interactions subsequent to their creation. Thus, antiprotons provide a unique channel for studying the proton cosmic-ray history. Furthermore, as will be discussed in later sections, the kinematics of production greatly suppresses the creation of low-energy antiprotons so that their expected spectrum differs dramatically from the slowly changing power laws of the other cosmic rays. Thus, the antiproton spectrum should be very sensitive to energychanging stochastic processes which may act upon the particles during propagation in interplanetary and interstellar space. If such processes occur, they could fill in this suppressed spectral region, while not noticeably altering power law spectra.

This paper describes a new balloon-borne experiment for measuring the cosmic-ray antiproton flux and extending the search for cosmic-ray antihelium. Instead of using the curvature in a magnetic field to separate matter from antimatter, this experiment identifies antimatter events by their annihilation radiation, in the same fashion as low-energy antimatter searches in emulsions (Apparao 1968). Residue from background processes which could masquerade as antimatter events has been reduced to a low level. The apparatus is capable of detecting antimatter between about 85 and $300 \mathrm{MeV}$ per nucleon. Particles of this low energy are likely to have experienced a significant energy change in the solar cavity due to the "adiabatic deceleration" resulting from interaction with the outgoing solar wind (Urch and Gleeson 1972, 1973). Although this complicates the interpretation of low-energy $\bar{P}$ measurements, it also raises the effective energy window of the experiment to a regime where $\bar{P}$ production is somewhat less suppressed by the production kinematics. On the other hand, antiprotons produced nearby in the residual atmosphere above the apparatus have not had the ben- efit of energy loss in the interplanetary region. At the energy of interest here, this eliminates the need of an atmospheric background subtraction from the measured data sample (Gaisser and Levy 1974).

\section{APPARATUS}

A detailed description of the apparatus and its calibration is presented elsewhere (Buffington et al. 1979; Pennypacker et al. 1979). ${ }^{3}$ For completeness, however, a brief discussion is included here. Previous antimatter searches have generally not been discriminating in their recording of data, depending instead upon subsequent analysis to perform the antimatter search. In this experiment, however, a $\bar{P} / P$ ratio of order $10^{-4}$ was anticipated, with only a few percent of the incident events falling within the energy range covered by the apparatus. Therefore, a selective trigger was devised to enrich the recorded data sample in potential antimatter candidates. Figure 1 shows a schematic representation of the apparatus, while Table 1 lists the detector thresholds and other specifications. Figure 2 presents a block diagram of the electronics.

To be recorded as a potential $\bar{P}$ candidate, an incident particle must generate a response in each of the four scintillators above fixed threshold settings and yet not exceed an additional threshold requirement for the Cerenkov counter. Each event record includes a spark chamber visualization of the event topology, and an oscilloscope recording of the pulses and their relative timing for each of the four scintillators and the Cerenkov counter. Each of two cameras operating simultaneously records the complete event information. An aluminum shell encloses the experiment to maintain sea level pressure during the balloon flight. Although some data are returned by telemetry, the detailed analysis requires recovery of the film. The bottom scintillator $\mathrm{S}_{4}$ could be removed from the trigger requirement by radio command, to permit recording of the proton flux as a cross-check of instrument performance.

From Figure 1, it is apparent that the fourfold scintillator coincidence requires penetration of the thick lead stack comprising the lower spark chamber. On the other hand, the velocity restriction imposed by the Cerenkov anticoincidence threshold, and by the thresholds for the top three scintillators for charge $Z=1$ particles, greatly limits the number of particles capable of reaching $S_{4}$.

\footnotetext{
${ }^{3}$ In some particulars the earlier treatment (Buffington et al. 1979) differs from that presented here. In the case of a disagreement, the authoritative information is that presented here. The most important changes are the correction of an error in the calculation of geometry factor, the inclusion in "detection efficiency" (which here is implicit in the geometry factor calculation) of the topological event selection criteria, as determined after the balloon flight, and scintillator threshold cutoffs. These three effects result in a factor of 10 reduction from the event collection estimates of the previous work.
} 

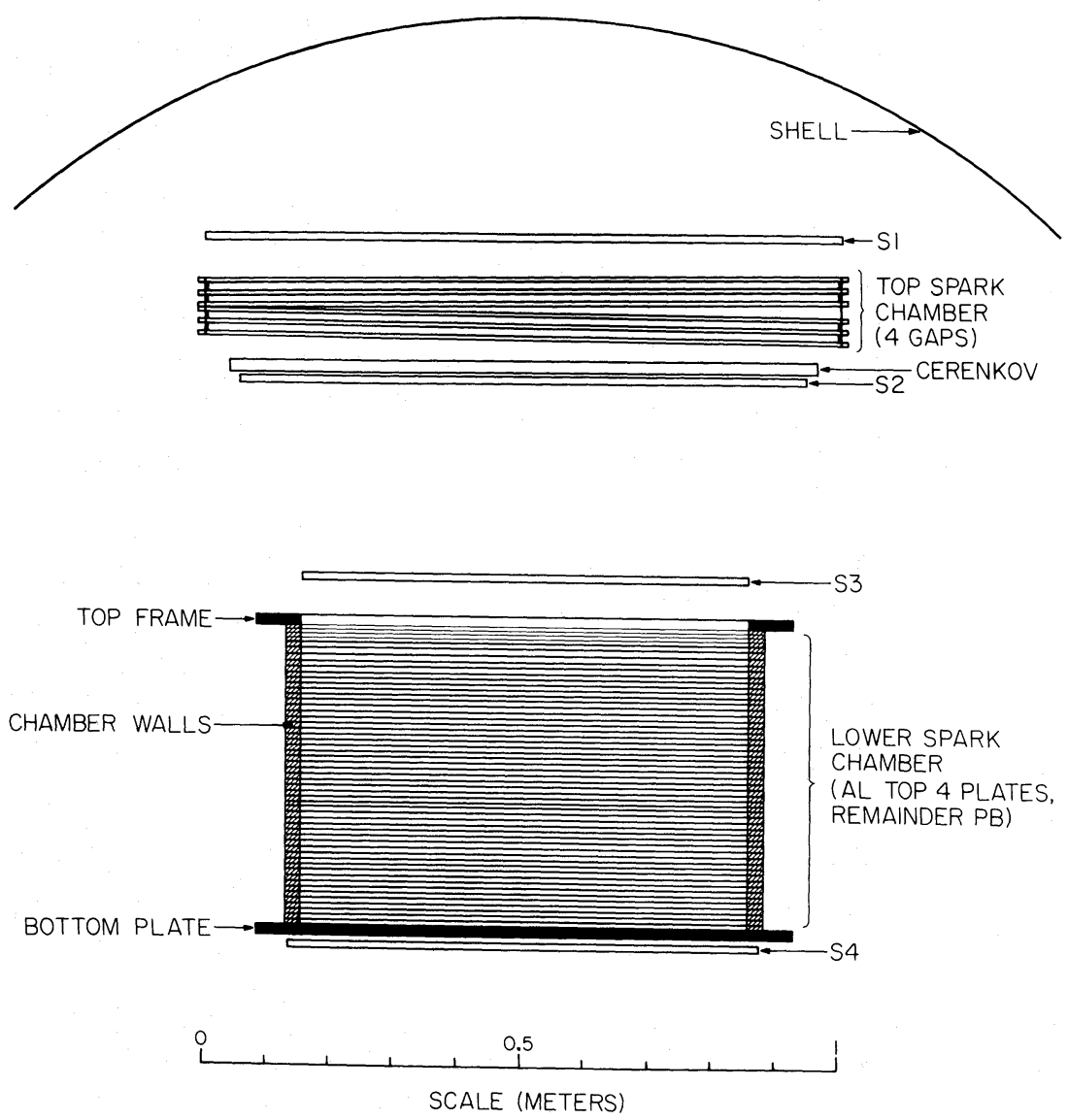

FIG. 1.-Schematic diagram of the apparatus. The trigger scintillators $\left(\mathrm{S}_{1} \rightarrow \mathrm{S}_{4}\right)$ and Cerenkov counter are plastic; the top spark chamber contains foam and aluminum; and the bottom spark chamber contains lead and aluminum. The steel plate beneath the lead chamber is 1.3 $\mathrm{cm}$ thick. The entire experiment is enclosed within two hemispherical shells which provide a pressurized environment for balloon flight. The shell is $2.4 \mathrm{~m}$ in diameter and is typically $0.7 \mathrm{~g} \mathrm{~cm}^{-2}$ thick.

TABLE 1

DETECTOR THRESHOLDS AND OTHER SPECIFICATIONS

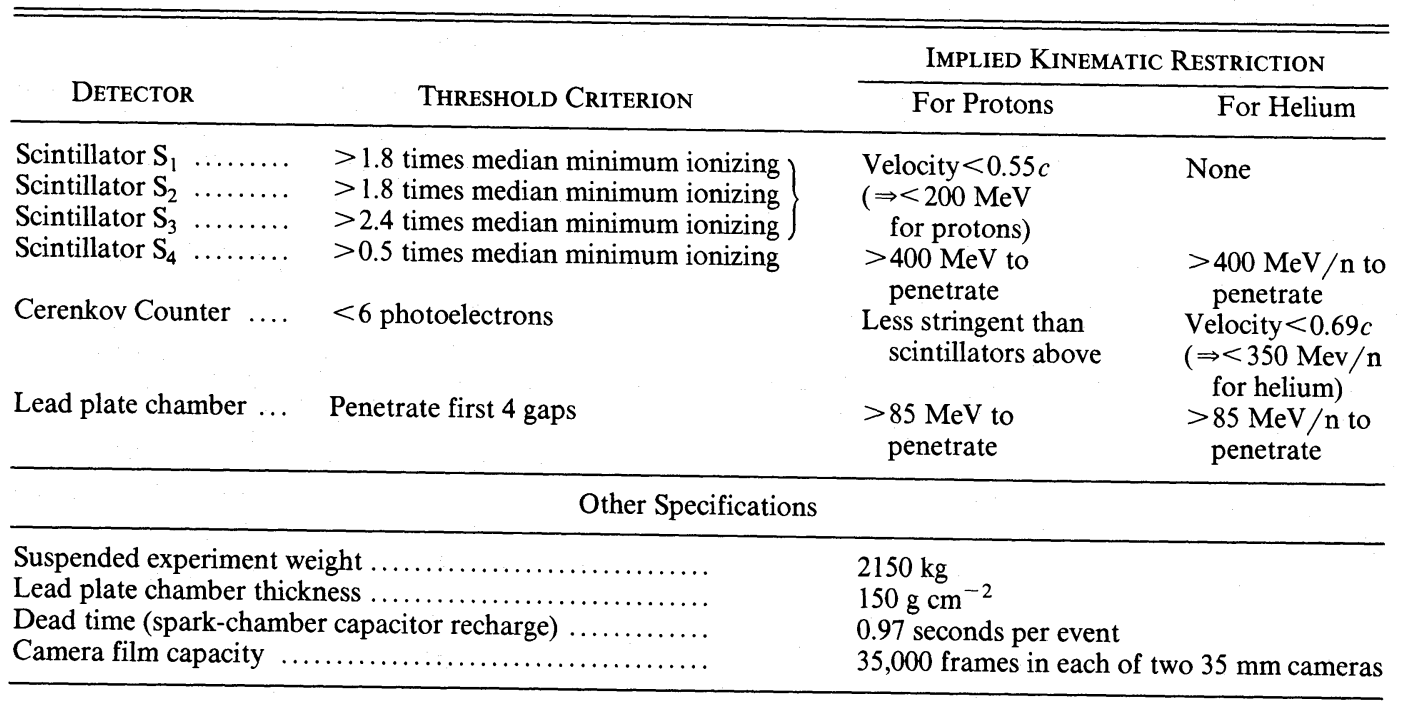




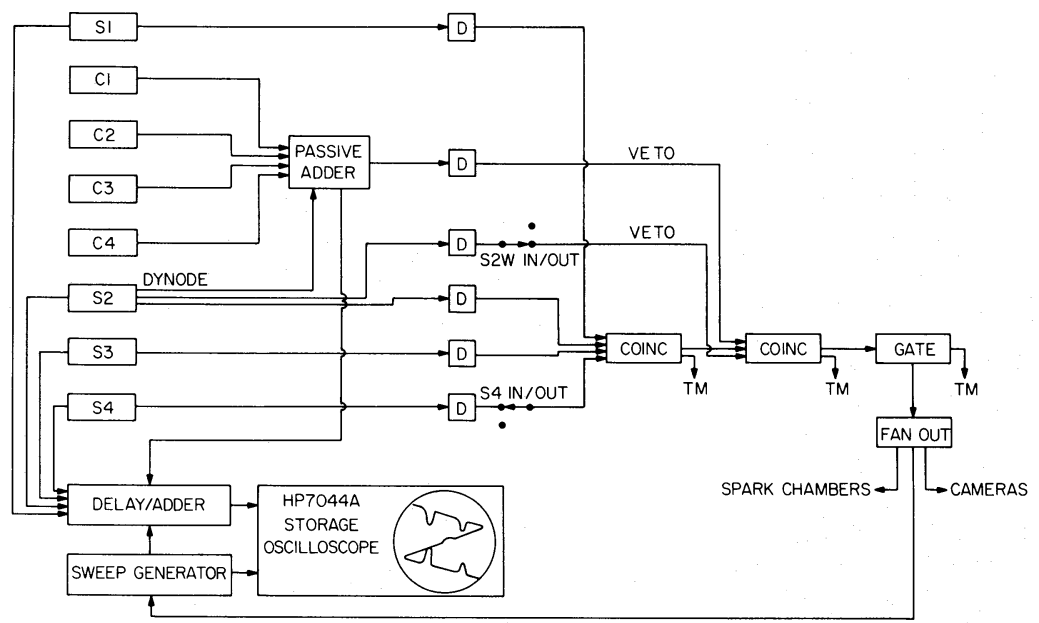

FIG. 2. - A schematic diagram of the electronics for the selective trigger. The scintillators $S_{1}$ through $S_{4}$ are actually viewed by two photomultipliers each, whose responses are added passively, with their outputs indicated here by individual boxes. Similarly, each box for the Cerenkov counter represents the outputs of six photomultipliers that have been added actively. The passive adder following these forms the 24-photomultiplier total output and subtracts a small portion of the $S_{2}$ signal to remove a scintillation contribution from the Cerenkov counter response. The symbol " $\mathrm{D}$ " indicates a discriminator, and "TM" indicates a telemetry monitor output. The gate imposes a $0.97 \mathrm{~s}$ dead time for spark chamber capacitor recharge. NIM level logic is used throughout. The four scintillator and Cerenkov signals are delayed relative to each other in $\sim 50 \mathrm{~ns}$ increments and are displayed on the oscilloscope using a Z-shaped baseline as indicated in the box above. Timing of individual pulses can be measured on the record of the oscilloscope trace to an accuracy of about $1 \mathrm{~ns}$.

Protons and noninteracting helium nuclei satisfying these threshold criteria can at most penetrate about threequarters of the way through the lead stack. Deuterons and tritons above 290 and $225 \mathrm{MeV}$ per nucleon, respectively, can penetrate to $S_{4}$, but only rarely can these particles satisfy the scintillator thresholds. In all, the trigger criterion allows approximately one in 1000 incident cosmic rays to trigger the apparatus. On the other hand, typically $8 \%$ of the antiprotons incident between 100 and $300 \mathrm{MeV}$ succeed in satisfying the trigger criterion because $\pi^{ \pm}$daughters from the annihilation reactions in the lead are able to penetrate and reach scintillator $\mathrm{S}_{4}$. We anticipated that annihilation pions traveling back through the Cerenkov counter might cause an unwanted rejection of antimatter events. To avoid this, the timing of the Cerenkov pulse was set relative to the scintillator pulses so the signals from such particles, typically delayed by $10 \mathrm{~ns}$, arrive too late at the coincidence circuit to reject the event.

Figure 2 shows a second discriminator (S2W) connected to scintillator $S_{2}$. The threshold is set at about 25 times the energy loss of minimum-ionizing protons for the purpose of rejecting potential triggers from nuclei heavier than helium. Since during the balloon flight the inclusion of S2W did not provide a significant reduction in trigger rate, the bulk of the data was taken without it. A small portion of the $S_{2}$ signal subtracted from the Cerenkov counter signal removes the scintillation contribution in the Pilot 425 radiator. This contribution was found to be $3 \%$ of the relativistic Cerenkov signal. Because the photomultiplier outputs from $S_{2}$ saturate for large pulses, this subtraction was likely ineffective for nuclei with charge $Z \gtrsim 4$, which may explain why the second discriminator on $\mathrm{S}_{2}$ made little difference in the trigger rate. Scintillation photons had already caused the high- $Z$ events to be rejected by the Cerenkov criterion, even though they were below the velocity threshold.

Lead was chosen for the lower spark chamber material because of its favorable ratio of $d E / d x$ to radiation length. The annihilation reactions also generate $\pi^{0}$ mesons. These decay to $\gamma$ rays, which shower and die out close to the vertex, thus permitting a clear visualization of the charged-prong annihilation topology in the spark chamber pictures. The top spark chamber provides an additional picture of the incident particle and a convenient means of recognizing and rejecting multiparticle events.

A quartz clock photographed with each event provides the experiment timing reference. An additional reference is obtained through telemetry, which provides a running tally of the outputs of the two coincidence circuits of Figure 2 and of the final gated trigger. The telemetry also provides a continuous monitor of discriminator counting rates, command states, and various housekeeping functions. A current indicator, microphone, and film counter monitor the performance of spark chambers and cameras.

The expected properties of the apparatus were calculated by a Monte Carlo program. The calculation used the range energy results tabulated by Janni (1966), the antiproton prong number distribution for $\bar{P}$-carbon annihilation measured by Agnew et al. (1960), the pion 
momentum distribution for $\bar{P}-P$ annihilations reported by Renner (1966), and the pion momentum range data appearing in Trower (1966). Daughter pions were generated isotropically. Inflight annihilations were Lorentztransformed to the instrument frame of reference. In-flight annihilations comprise as much as one-third of the events for $300 \mathrm{MeV}$ antiprotons. Even so, the fraction of in-flight annihilations in the data should be typically only $15 \%$, and their inclusion in the geometry factor calculation makes a difference of only about $10 \%$. Agnew et al. (1960) and Ekspong et al. (1961) discuss some observed differences between $\bar{P}-P$ and $\bar{P}$-heavy nucleus annihilations. They find that typically one or more "black" prongs (slow, heavily ionizing protons) accompany the $\pi$ mesons due to the rescattering of annihilation daughters within the nucleus. It is rare, however, that these extra prongs have the $\gtrsim 100 \mathrm{MeV}$ necessary to be recognizable in the lead plate spark chamber, and they are therefore ignored in the calculation.

The scintillator threshold requirements imposed on the Monte Carlo results were scaled from ground muon calibrations using the appropriate $d E / d x$ values. Topological requirements identical to those imposed upon the data were applied in the calculation: at least three charged prongs must be discernible in the lead plate chamber, each penetrating at least three spark chamber gaps and at least one reaching $\mathrm{S}_{4}$; in the case of only three prongs, at least one must travel upward. When an upward prong passed through scintillator $S_{3}$, as expected for typically half of the events, the $S_{3}$ threshold requirement was considered satisfied. Figure 3 shows the resulting geometry factor as a function of incident energy. The topological selection is implicit in the geometry factor calculation, rather than being calculated as a separate "event selection efficiency." Figure 3 also shows the geometry factor for antiprotons that would have been achieved if it were possible to include all antiprotons satisfying the trigger requirement. A separate calculation used a momentum and energy-conserving model for $\bar{P}-P$ annihilations (Vandernueler and Trippe 1978, private communication) instead of merely imposing the average momentum distribution upon the prong distribution. This calculation agreed with the results presented here to within about $10 \%$. We feel this is a reasonable estimate of the potential systematic error in the geometry factor calculation due to the uncertainty in parameterization of the annihilation dynamics. The integral geometry factor for antiprotons can be approximated as $0.012 \mathrm{~m}^{2}$ sr between 85 and $300 \mathrm{MeV}$, although in fact the instrument's sensitivity extends above this range.

\section{MOUNTAINTOP CALIBRATION}

The selective trigger described in the previous section was successful at rejecting most background sea level cosmic-ray events. However, the sea level trigger rate

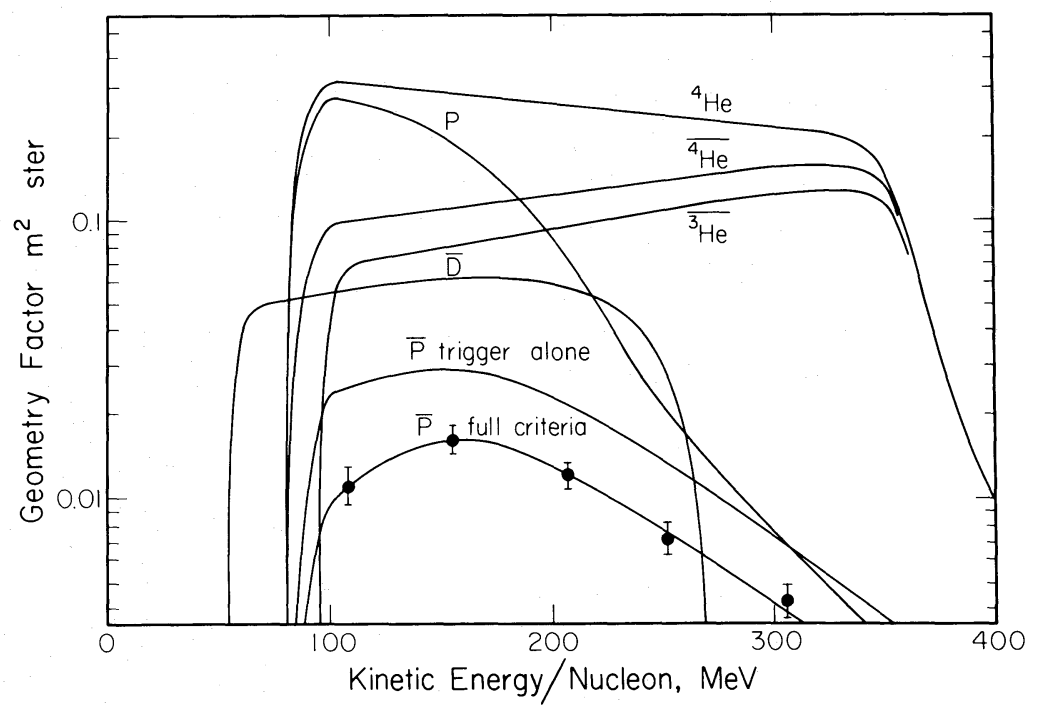

Fig. 3.-A plot of the Monte Carlo calculated geometry factor as a function of incident energy at the top of the apparatus for various particles and antiparticles. The results shown include all trigger and topological identification criteria, except for the higher of the two $\bar{P}$ curves, which is for the trigger alone. The sharp cutoff at low energy for each particle is caused by the requirement that all particles penetrate at least to the fifth gap of the lower spark chamber. Curves for more complex antimatter are higher than those for antiprotons because the larger number of annihilation daughters increases the chance that one of them can penetrate through to $S_{4}$. The high-energy cutoffs are caused by the scintillator thresholds for protons and antiprotons, by the Cerenkov counter for helium and antihelium, and by penetration through the lead plate spark chamber for antideuterons. The $\bar{P}$ and $\bar{D}$ curves rise above the $P$ curve at higher energy because annihilation daughters raise the expected energy deposition in $\mathrm{S}_{3}$. The "data points" on the lower $\bar{P}$ curve show the Monte Carlo calculation results from which that curve was deduced. 
was only one per hour, too slow to be practical for studying the various non-antimatter-induced triggers. We expected that these background triggers were initiated by strongly interacting particles, primarily protons, since the trigger criterion was most efficient at rejecting muons and electrons. Therefore we moved the apparatus to the New Mexico State University's mountaintop laboratory at Sacramento Peak, New Mexico. Here, at a residual atmospheric depth of $730 \mathrm{~g} \mathrm{~cm}^{-2}$, the proton flux at several hundred $\mathrm{MeV}$ was increased approximately tenfold from sea level, and a corresponding increase occurred in the background trigger rate.

The primary objective was a verification that the non-antimatter processes satisfying the trigger criterion could be easily distinguished from antimatter events, by examining the spark chamber topology. The most common topology recorded showed a slow proton stopping in the lead, legitimately satisfying the trigger criterion except in scintillator $S_{4}$. The observed trigger rate for this type of event is explained by $5 \%$ of the protons scattering a neutron from a lead nucleus as they are slowing down, and then $2 \%$ of these neutrons interacting in $S_{4}$ to complete the trigger requirement. This topology was easily distinguishable from that of an antiproton meeting our selection criteria, since it lacked the charged $\pi^{ \pm}$annihilation prongs, and no particle was seen to exit the bottom of the lead plate spark chamber. Other background topologies included multiparticle events and apparent interactions in nearby gondola material such as the lead plate spark chamber frames. All of these were readily distinguishable from the expected antimatter topology. In 55 hours of data at the mountaintop, the apparatus recorded at most a single event exhibiting an acceptable antiproton topology. We say "at most" because the oscilloscope information was not available for these data, and this event might have been rejected on that basis. Using the results of Barber (1976), we expected a $\bar{P}$ flux at the mountaintop of about $2 \times 10^{-6} \overline{\mathrm{P}}\left(\mathrm{m}^{-2} \mathrm{sr}^{-1} \mathrm{~s}^{-1}\right)$, or $5 \times 10^{-3}$ events in the complete mountaintop exposure. The calibration also yielded information about the sensitivity of trigger rate to the experiment threshold settings; these details are presented elsewhere (Pennypacker et al. 1979).

\section{BALLOON FLIGHT}

Since the interaction mean free path in the atmosphere is about $50 \mathrm{~g} \mathrm{~cm}^{-2}$ for antiprotons and about 30 $\mathrm{g} \mathrm{cm}^{-2}$ for antihelium, the apparatus must be flown above most of the atmosphere to reduce attenuation of the particle flux. In addition, since the energy window for identifying these particles extends from 85 to 300 $\mathrm{MeV}$ at the apparatus, the experiment must take place at a sufficiently low geomagnetic cutoff that such lowenergy particles are not excluded. The launch site chosen was The Pas, Manitoba, Canada. A desired altitude of $29 \mathrm{~km}$, or $9 \mathrm{~g} \mathrm{~cm}^{-2}$ of residual atmosphere, represented a compromise between atmospheric attenuation and flight duration, since higher altitudes tend to have greater wind velocities. Taking into account energy loss in the atmosphere, the instrument covers 130 to 320 $\mathrm{MeV}$ for antiprotons and 130 to $370 \mathrm{MeV}$ per nucleon for antihelium at the top of the atmosphere.

The instrument was flown on 1980 June 18. Table 2 presents some pertinent information about the flight. During the data taking, the calculated vertical geomagnetic cutoff varied from 0.60 to $0.77 \mathrm{GV} / c$ (Shea, Smart, and Carmichael 1976). Much of the anticipated data might have been lost if these values were correct, since they correspond to antiproton kinetic energies of 175 and $280 \mathrm{MeV}$ respectively. However, Fanselow and Stone (1972) have reported a disagreement between measured and calculated invariant cutoff latitudes of $3^{\circ}-5^{\circ}$ at lower proton energies. If only a 1.5 error in the calculation persists at our latitudes, the cutoff values at beginning and end of flight are lowered to 0.46 and 0.61 $\mathrm{GV} / c$, corresponding to 110 and $180 \mathrm{MeV}$ energy cutoffs, which would have only a small impact on the data. No evidence for any significant geomagnetic cutoff effect appears in the $\bar{P}$ data.

TABLE 2

FLIGHT INFORMATION

\begin{tabular}{|c|c|}
\hline Launch & Time: $0821 \mathrm{CDT}, 1980$ June 18 . Location: $53^{\circ} 44^{\prime} \mathrm{N}, 101^{\circ} \mathrm{W}$. \\
\hline Float & $\begin{array}{l}\text { Reach } 28.5 \mathrm{~km} \text { at about } 1000 \mathrm{CDT} \text {, steady altitude for } 14 \frac{1}{2} \mathrm{hr} \text {, then drop to } 23 \mathrm{~km} \text { for } \\
\text { another } 4 \frac{1}{2} \mathrm{hr} \text {. }\end{array}$ \\
\hline Vertical residual atmosphere $\ldots$ & $9 \mathrm{~g} \mathrm{~cm}^{-2}\left(14 \frac{1}{2}\right.$ hours $)+$ average $18 \mathrm{~g} \mathrm{~cm}^{-2}\left(4 \frac{1}{2} \mathrm{hr}\right) ;$ average $11 \mathrm{~g} \mathrm{~cm}^{-2}$ for whole flight \\
\hline Recovery $\ldots \ldots \ldots \ldots \ldots \ldots \ldots$ & Impact: near Vermilion, Alberta. Location: $53^{\circ} 12^{\prime} \mathrm{N}, 111^{\circ} \mathrm{W}$. \\
\hline Data taking . & $\begin{array}{l}20,562 \text { data frames with full trigger criterion; } 1,750 \text { data frames with } \mathrm{S}_{4} \text { removed from } \\
\text { trigger criterion }\end{array}$ \\
\hline $\begin{array}{l}\text { Duration (for full antimatter } \\
\text { trigger criterion) } \ldots \ldots \ldots \ldots \ldots\end{array}$ & $18.4 \mathrm{hr}$ at float; $4.6 \times 10^{4} \mathrm{~s}$ of live time \\
\hline
\end{tabular}


V. DATA ANALYSIS

\section{a) Data with $\mathrm{S}_{4}$ Not Included in the Trigger Criterion}

As Table 2 shows, $8 \%$ of the events recorded had $\mathrm{S}_{4}$ excluded from the trigger criterion. These events are expected to be low-energy protons generated as secondaries in the residual atmosphere above the experiment, together with some primary protons and helium. The match between these data and the expected fluxes provides a check on the operation of the apparatus.

The live time for the major portion of the experiment is determined by subtracting the dead time per event, multiplied by the number of events, from the total time. The trigger rate for the data without $\mathrm{S}_{4}$, however, was about 170 times greater than the rate with $S_{4}$ included in the trigger criterion, and the live time was only about $10^{-2}$ of the clock time (approximately 30 minutes). Therefore the trigger rate for this portion of the data is inferred from the telemetered coincidence circuit outputs, which were accurate only to $\pm 20 \%$.

The trigger rate without $S_{4}$ was $75 \pm 15$ events per second. About $85 \%$ of these events have the expected topology of a particle entering the apparatus from above. The other $15 \%$ typically have several particles present at once, no one of which appears to satisfy the detector geometry requirement. For events with a proper topology, one-third have evidence of an accompanying particle, usually penetrating only the thin top spark chamber. Calculations indicate that about $10 \%$ of the events ought to have such an accompanying particle produced in the nearby gondola material. The remainder are presumably due to a chance passage within the spark chamber resolving time, of an uncorrelated, usually low-energy particle. A similar fraction of extra tracks also occurs for the data with $\mathrm{S}_{4}$ included in the trigger criterion.

About four-fifths of the events with a particle incident from above have the particle entering the top of the lead plate spark chamber; about two-thirds of these penetrate beyond the first four gaps. These more penetrating events, typically particles with greater than $85 \mathrm{MeV}$ per nucleon at the top of the apparatus, provide a measurement of the energy spectrum for protons up to about $200 \mathrm{MeV}$ and of helium up to about $350 \mathrm{MeV}$ per nucleon. Protons and helium nuclei are distinguished by the pulse height of scintillator $\mathrm{S}_{3}$, as recorded by the oscilloscope. The penetration of each event, together with its incident angle, provides a measure of the range and hence energy for the particle. The subsequent analysis includes corrections for inelastic nuclear reactions in the lead, transferring events to an apparent lower energy bin (Janni 1966) and a factor of 1.18 attenuation correction for particles interacting above the fourth gap of the lead plate spark chamber. Figure 4 shows the resulting spectra.

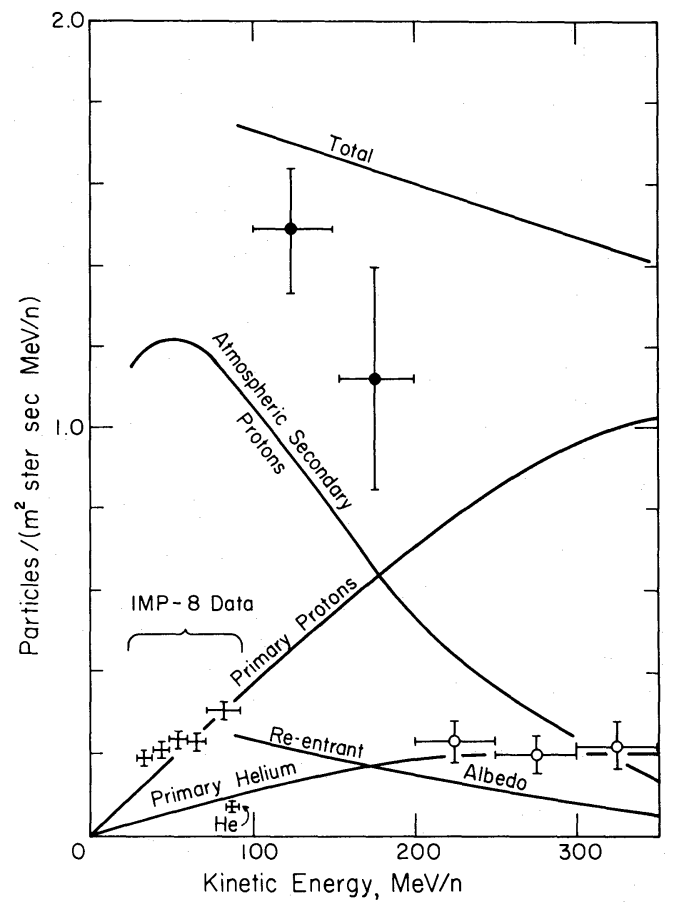

Fig. 4. - A plot of measured and expected particle fluxes at the top of the apparatus: closed circles, protons + helium; open circles, helium alone. Errors are statistical only; there is also an overall scale factor uncertainty of $20 \%$. The IMP 8 data were measured just before the balloon flight. The primary proton curve is chosen to pass through these points. The primary helium curve is chosen to fit best the IMP 8 helium measurement and the three helium points measured in this experiment. Atmospheric secondaries are from Rygg and Earl (1971), and the reentrant albedo is from Ormes and Webber (1968).

Also shown in Figure 4 are the expected fluxes from atmospheric secondaries, reentrant albedo, and primary protons and helium. The proton flux is extrapolated (see, for example, Urch and Gleeson 1972, for expected spectral shapes near solar maximum) from IMP 8 satellite measurements of protons and helium, for the time period from 1980 June 11 through 1980 June 18. These were kindly provided by Roger Pyle of the University of Chicago (private communication). The reentrant albedo flux is taken from Ormes and Webber (1968), under the assumption that the albedo is not significantly different between The Pas, Manitoba, and Ely, Minnesota. The atmospheric secondary flux is scaled from Rygg and Earl (1971) for a daily average Deep River neutron monitor rate of 6368 per hour at the time of our flight (M. Kawerninski 1980, private communication).

Within statistics and the $20 \%$ normalization error resulting from uncertainty in the trigger rate, agreement between the expected and observed proton and helium fluxes is satisfactory, although a smaller primary proton flux would give a better fit. Because of the large contribution of secondary protons, the instrument does not 
provide a direct measurement of the proton flux, and we instead employ the extrapolation of the IMP 8 measurements. The IMP 8 proton flux, in correlation with the neutron monitor, appears to be larger by a factor of 2 , when this solar cycle is compared with the previous one (Van Hollebeke, Wang, and McDonald 1972). On the other hand, the helium flux and neutron monitor correlation agrees with the previous cycle. Later in this paper, in calculating a $\bar{P} / P$ ratio and setting an upper limit on the $\overline{\mathrm{He}} / \mathrm{He}$ ratio, we use the primary fluxes of Figure 4. Although the helium flux is accurate to about $20 \%$ and is directly measured in this experiment, the inferred proton flux comes entirely from the extrapolation of the IMP 8 data. Our measurement here, and a comparison with modulation effects associated with the previous solar cycle, suggest the value chosen may be too large by a factor of up to 2, but we feel the extrapolation of the IMP 8 flux is the best choice at present.

\section{b) Data with $S_{4}$ Included in the Trigger Criterion}

These data, expected to be enriched in potential antimatter candidates, were the main objective of the balloon flight. The topological composition of these events was found to vary with time only slightly throughout the flight. Most of the events have the same character as those observed on the mountaintop-a single particle stopping in or passing through the lead $25 \%$ of the pictures) or two or more energetic particles simultaneously incident $(50 \%)$. However, the flight data differ significantly from the mountaintop data in that $15 \%$ of the events consist of a single penetrating particle with a kink in its trajectory, or a branch into two tracks, usually with a narrow angle between two forward going prongs. About $1 \%$ of the events have the particle branching into three forward going prongs. The $\mathrm{S}_{3}$ scintillator pulse height shows that $95 \%$ of these events had a helium nucleus incident, which explains their absence at the mountaintop, since the helium flux there is small. The three-prong events that exhibit a small pulse in $S_{3}$ we interpret as being generated by deuterons or tritons created in helium fragmentations in or above $\mathrm{S}_{3}$. Unfortunately, such interactions occurring within the apparatus cannot all be identified because the pulses from scintillators $S_{1}$ and $S_{2}$ are clipped, and only their timing is preserved.

A small fraction of the fragmentation reactions in the spark chamber are worrisome because they were found to exhibit a topology similar to that expected from antiproton annihilations. This background was not anticipated in the original conception of the experiment. Even though $95 \%$ of these fragmentations can be rejected as helium nuclei by the $S_{3}$ pulse height, the oneand two-prong topologies are sufficiently plentiful in the residual data that it is not possible to separate the residue from one- or two-prong antiproton annihilations. Even with the requirement of three or more prongs, a background of 10 to 15 events is expected, based on the remaining $\sim 5 \%$ of the one- and two-prong events that satisfy the small $S_{3}$ pulse height requirement. However, the great majority of these events have all prongs in the downward direction. This is why we impose on the final antimatter selection a topological requirement that there be three or more prongs, with one upward in the case of only three prongs, for an event to be considered as an antimatter candidate. Even this requirement does not guarantee the complete removal of this background, since a fragmentation reaction between a deuterium or tritium nucleus and a target lead nucleus can give rise to "gray" tracks in the spark chamber, due to $\gtrsim 100 \mathrm{MeV}$ protons produced in the nuclear cascade induced by the reaction (Metropolis et al. 1958; § 12-4-d in Powell, Fowler, and Perkins 1959). Usually the fragments of the interacting beam particle and these gray secondaries travel forward in the laboratory frame (target at rest), but a few percent of them are in the backward hemisphere. The removal of this background, however, results in the reduction by a factor of 2 of the antiproton geometry factor, as shown in Figure 3.

Initially, scanners were instructed to examine each recorded event, listing any which might have a topology characteristic of antimatter annihilations: an incident track which connects to three or more straight or kinked prongs in the lead plate chamber. These criteria were significantly less stringent than the final ones imposed on potential antimatter events. This technique reduced the number of events while retaining a high scanning efficiency and allowed the authors to impose the more stringent criteria. All of the data were scanned twice. About 1500 events were listed by the scanners for more detailed examination. Most proved to have either more than one energetic particle present or some other topological uncertainty which the scanners felt deserved further analysis. Out of the 1500 events originally listed, 64 were found that satisfy all of the following topological requirements:

1. A particle apparently enters the lead plate chamber from above and penetrates at least as far as the fourth gap. A corresponding track is visible in the top spark chamber.

2. An interaction occurs within the lead plate chamber in which the incident particle makes at least three outgoing prongs, one of which passes through the lower scintillator $\mathrm{S}_{4}$. All prongs penetrate at least three spark chamber gaps.

3. In the case where only three prongs are present, at least one travels upward in the lead plate spark chamber.

Figure 5 is an example of an event that satisfies these criteria (and also passes the further selection below).

We expect that some events might be caused by a high-energy particle, above the multipion production threshold, incident upon the lead plate chamber from 

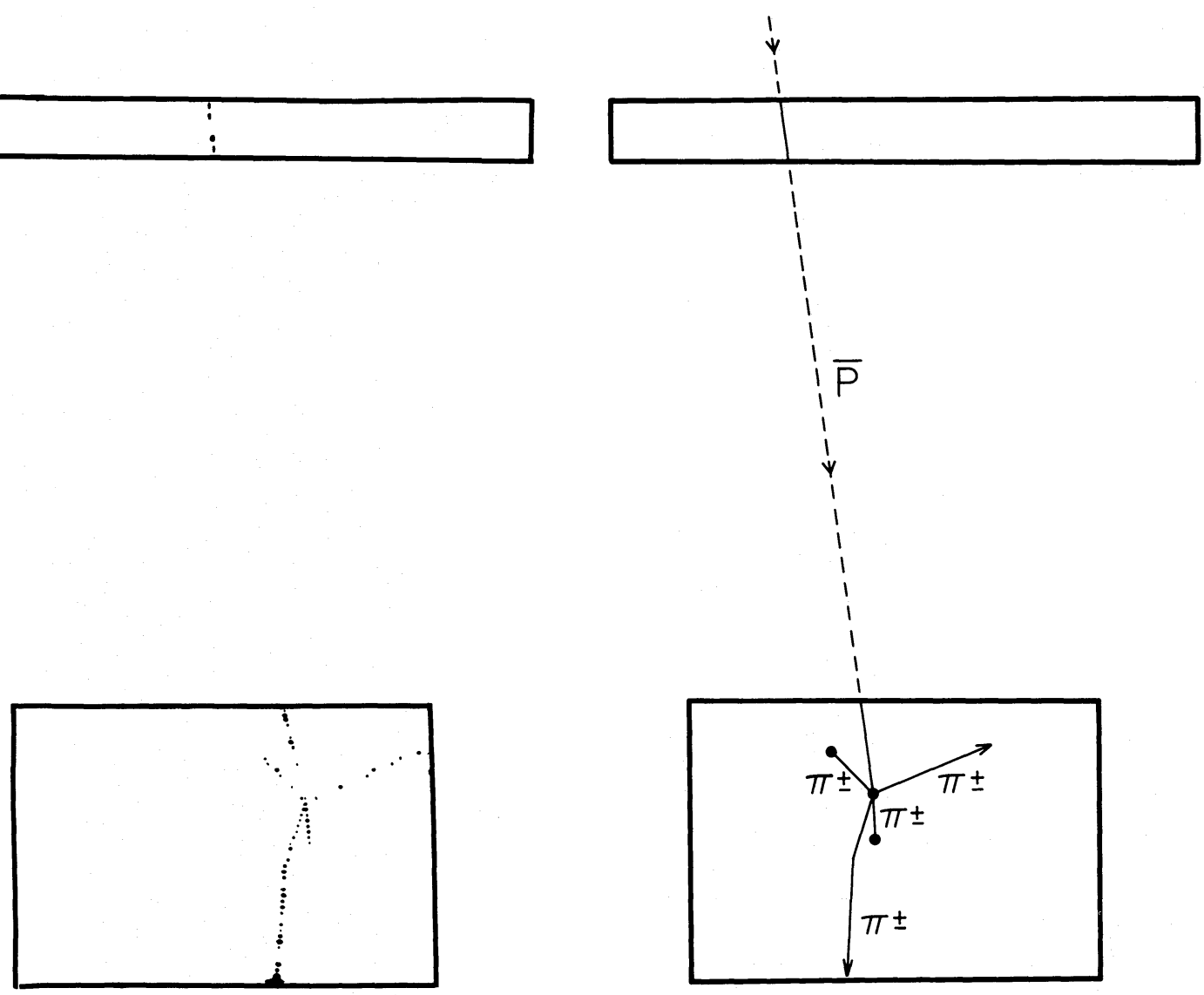

FIG. 5.-Example of an antiproton annihilation. To the left are shown the sparks marking the event topology, with the flight optical format rearranged to correspond to the apparatus (Fig. 1) and with two unassociated tracks and a few random sparks removed. To the right is a tracing of the topology inferred from the sparks. Two of the daughter pions stop within the chamber, one escapes out the side, and the fourth scatters and escapes out the bottom, where it passes through scintillator $\mathrm{S}_{4}$. These pions together deposited at least $450 \mathrm{MeV}$ of energy in the spark chamber, neglecting their masses and whatever kinetic energy was carried away by the two escaping particles.

the side. This could result in a multiprong interaction topology in the chamber, with the trigger criterion being met entirely by secondary particles. To remove this background, we measured the timing of scintillator $\mathrm{S}_{3}$ relative to that of $S_{1}$ on the oscilloscope record. Sideinduced events are expected to have $S_{3}$ early compared with $S_{1}$, while events with a slow downward going particle typically show $S_{3}$ delayed relative to $S_{1}$ by 6-13 ns. Figure $6 a$ shows the time difference $\left(t_{\mathrm{S}_{3}}-t_{\mathrm{S}_{1}}\right)$ distribution for a sample of events with the topology of a single particle penetrating through to $S_{4}$ without any indication of an interaction. These events are mostly helium, as determined by the $S_{3}$ pulse height, with $\sim 300$ $\mathrm{MeV}$ per nucleon, which underwent a proton-stripping reaction into a more penetrating triton or deuteron near the top of the lead plate chamber, which in turn, took typically $6 \mathrm{~ns}$ to pass between $S_{1}$ and $S_{3}$. These events have been used to determine the proper location of zero time, which has been placed $6 \mathrm{~ns}$ to the left of the peak in Figure $6 a$. Figure $6 b$ shows the timing distribution for the events satisfying all of the antimatter topology requirements. The timing distribution has two peaks, one corresponding to the normal top-induced events, and another with the $S_{1}$ pulse occurring later than the $S_{3}$ pulse. We have eliminated the 28 events in the left-hand peak of Figure $6 b$, attributing these to side-induced interactions, as opposed to a valid antimatter annihilation. All but two of these events show two or more upward prongs, one of which does not pass through the top part of the apparatus, and which could therefore have been the incoming parent particle.

Of the remaining 36 events, 19 are helium-induced, based on the $S_{3}$ pulse height analysis. The 17 remaining events meet all antiproton criteria. The velocity requirement imposed by the scintillators and Cerenkov counter guarantees that the parent particles are sufficiently below the multipion production threshold that protons, deuterons, and tritons do not generate the multiprong topology by this means. Decays of strange particles created above the apparatus and traveling down into the lead do not meet the topology requirements. Antiprotons provide the only reasonable explanation for these 


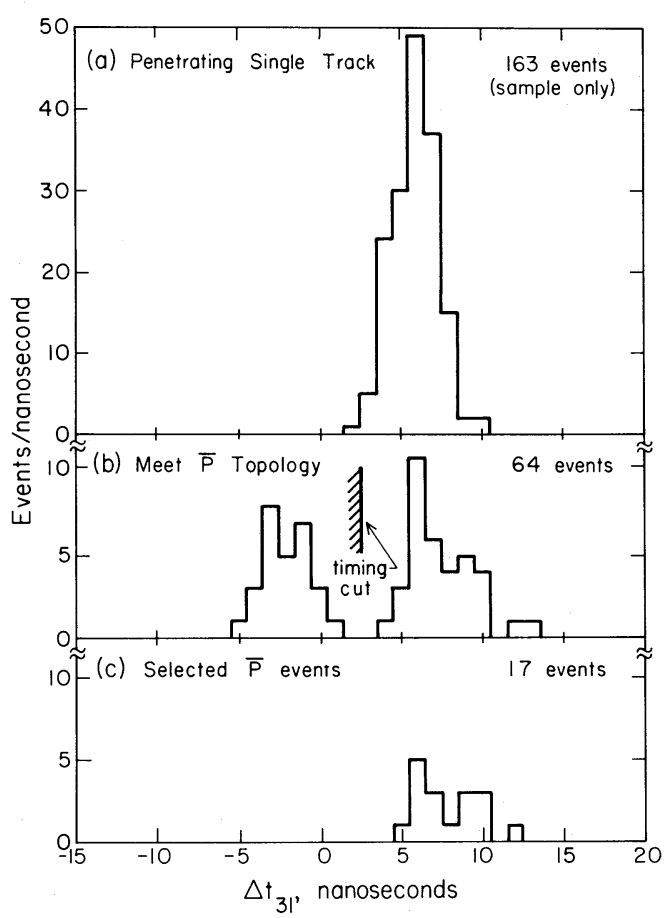

FIG. 6.-Distribution of the difference in time between scintillators $S_{3}$ and $S_{1}$ for two data samples. ( $a$ ) Helium events whose timing is assumed to be $+6 \mathrm{~ns}$. (b) Antimatter candidates which satisfy all of the topological requirements. Events with $\Delta t_{31}<2.5$ ns have been eliminated from consideration as antimatter and are attributed to the side-induced background process described in the text. (c) Events with small $S_{3}$ pulse height that constitute the $\bar{P}$ sample selected by this experiment.

events. Residual contamination by fragmentation, single pion production, and nuclear cascades in deuteron- and triton-induced events is estimated to be about one event. This estimate is based on the 5\% fraction of small $\mathrm{S}_{3}$ pulses seen in the one-prong, two-prong, and three-prong (all forward) events, times the 19 events that were rejected as helium-induced.

One might consider various background processes involving incident particles above Cerenkov threshold which were not rejected because of photoelectron fluctuations. All but three of the antiproton events had fewer than three photoelectrons in the Cerenkov pulse, less than half of the threshold requirement. If there were an important background process that was marginal in satisfying the Cerenkov criterion, the background events would cluster just below the threshold value. Typically half of the rejected helium events and three-prong (all forward) events with small $S_{3}$ pulse did this. On the other hand, the three antiproton events with more than three photoelectrons are about the number expected from a proper photoelectron distribution for these particles. Therefore, the Cerenkov pulse distribution is best consistent with only one or two events remaining in the $\bar{P}$ sample that could have come from a process that was marginal in satisfying the Cerenkov criterion, as deduced above.
There are three additional cross-checks which are consistent with the conclusion that most fragmentation reaction contributions have been successfully removed from the antiproton data sample. These are shown in Figure 7, where the prong number, pion energy deposition, and isotropy distributions are compared with the
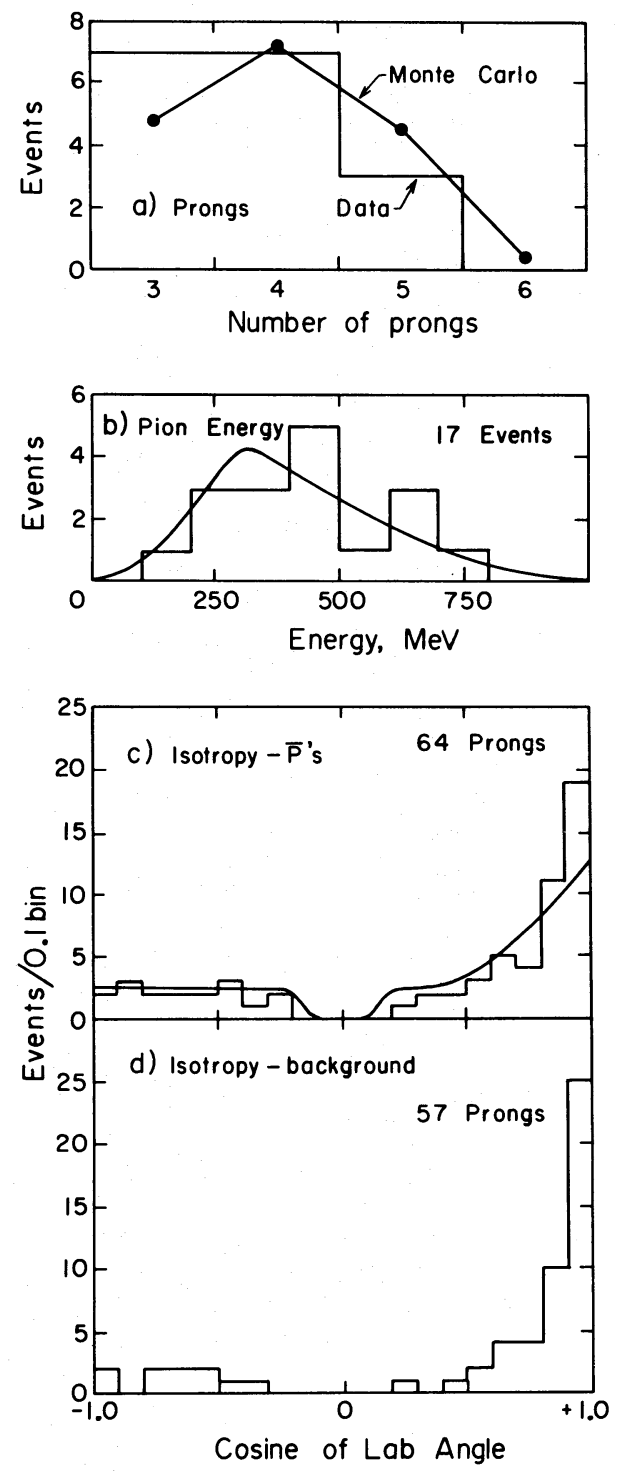

FIG. 7.- Results of various cross-checks upon the selected antiproton events. The data (histograms) are compared with predictions of the Monte Carlo calculation (smooth curves). (a) Number of observed secondary prongs. (b) Lower limit on secondary energy in the charged daughter pions, as deduced by lengths of the prongs in the lead plate spark chamber. For lower limit, escaping particles are assumed to have stopped immediately upon their exit from the chamber. (c) Distribution of prong angle relative to vertical. The "notch" at cosine $=0$ is caused by poor efficiency for recognizing tracks nearly parallel to the spark chamber plates. The rise in the forward direction is caused by the trigger requirement that one of the prongs pass through scintillator $S_{4} \cdot(d)$ As in Fig. $7 c$, but for events identified as helium-induced fragmentation reactions by the $S_{3}$ pulse height. 
predictions of the Monte Carlo program. The pion kinetic energy distribution is calculated on the assumption that all daughter prongs are pions and that escaping particles carry away no kinetic energy. Thus, this is a lower limit on the pion kinetic energy. The events which were rejected as helium-induced are about equally divided between those with three and those with four prongs with none having five prongs, and which have a pion energy distribution peaked at slightly lower energy. Figure $7 d$ shows the angular distribution for 16 of these events. The two distributions do not differ greatly, although the helium-induced events appear to have a greater fraction of the prongs in a forward direction. Figures $7 c$ and $7 d$ appear consistent with one or two fragmentation reaction events remaining in the antiproton sample, as predicted above. In the next section, for calculating the flux, one event is removed to take account of the possible residual fragmentation contamination. Two events are also removed because four antiprotons had an accompanying particle incident on $\mathrm{S}_{3}$ from above, the result of interactions in the upper gondola material. These extra particles helped satisfy the trigger threshold in $\mathrm{S}_{3}$, but they were not considered in the geometry factor calculation (Fig. 3). The average efficiency for these events was thereby raised by about a factor of 2 . Thus, the observed number of antiprotons for flux calculation purposes is reduced to 14 .

Because the dimmer appearance of the sparks in an event with many prongs might have caused the exclusion of some events, we force-developed the second roll of film to enhance possible dim sparks. No further antimatter candidates were found. More important, no events show the larger number of prongs that would characterize about half of the potential antideuterium events and all heavier antinucleus events.

\section{RESULTS}

\section{a) Antihelium}

The absence of antihelium candidates sets an upper limit on their flux ( $95 \%$ confidence level, which corresponds to three events) of $5 \times 10^{-4}$ antiparticles $\mathrm{m}^{-2}$ $\mathrm{sr}^{-1} \mathrm{~s}^{-1}$ for the energy range shown in Figure 3. Because an antideuteron might, half of the time, have six or fewer prongs showing in the lead plate chamber, the upper limit is $2 \times 10^{-3} \overline{\mathrm{D} \mathrm{m}} \mathrm{m}^{-2} \mathrm{sr}^{-1} \mathrm{~s}^{-1}$. Figure 8 shows the result for antihelium, presented in ratio to the helium flux [an average value of $0.18 /\left(\mathrm{m}^{2} \mathrm{sr} \mathrm{s} \mathrm{MeV} \mathrm{per}\right.$ nucleon), from Fig. 4], and including factors of 1.3 and 1.5 for attenuation in the apparatus and atmosphere. The value is $2.2 \times 10^{-5}$, the smallest ratio reported to date by a search for cosmological antimatter. Also shown are limits set for this ratio by previous experiments.

\section{b) Antiprotons at the Apparatus}

The 14 antiprotons observed in this experiment give a flux of 0.030 particles $\mathrm{m}^{2} \mathrm{sr}^{-1} \mathrm{~s}^{-1}$ at the top of the

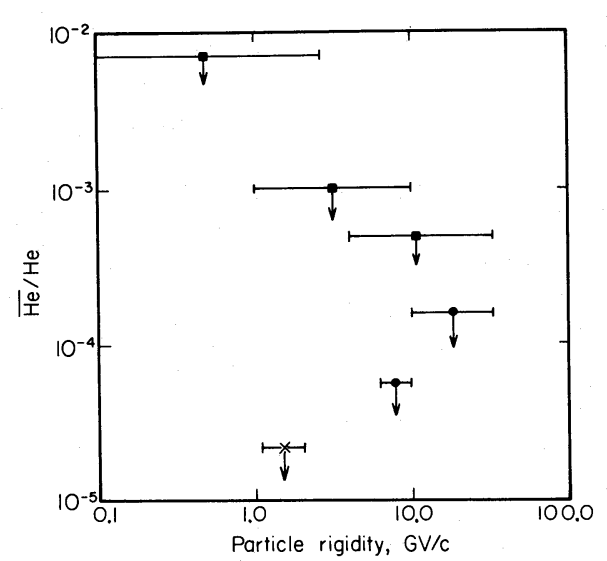

FIG. 8.-95\% confidence level upper limits on the ratio of antihelium to helium in the cosmic rays, as measured at $1 \mathrm{AU}$. Most data are summarized by Steigman (1976) (squares), but some data (circles) are from Badhwar et al. (1978), and the cross is the new measurement reported here.

apparatus between 85 and $\sim 300 \mathrm{MeV}$, which includes an attenuation correction factor of 1.2 to account for interaction of antiprotons in the $1.2 \mathrm{~g} \mathrm{~cm}^{-2}$ of aluminum and $4.6 \mathrm{~g} \mathrm{~cm}^{-2}$ of plastic above the fifth gap of the lead plate spark chamber. Because in-flight antiproton annihilations may shorten the apparent range of a portion of the antiproton sample, a spectrum cannot be extracted, and instead all of the data are taken together to yield a single differential measurement. The differential flux is $1.4 \pm 0.4 \times 10^{-4}$ antiprotons $\mathrm{m}^{-2} \mathrm{sr}^{-1} \mathrm{~s}^{-1} \mathrm{MeV}^{-1}$.

\section{c) Atmosphere}

The $11 \mathrm{~g} \mathrm{~cm}^{-2}$ average vertical residual atmosphere above the instrument both attenuates the cosmic antiproton signal and can potentially add a few extra antiprotons created in collisions between high-energy cosmic rays and the air nuclei. The attenuation correction factor which results from an assumed mean free path in air of $50 \mathrm{~g} \mathrm{~cm}^{-2}$ is 1.25 . To find the number of extra antiprotons generated in the atmosphere, we make use of the calculation of Stephens (1980) for the production rate of antiprotons in $P-P$ collisions. To convert his tabulated results to the proper value of residual atmosphere we multiply by $\left(\xi_{N} \mathrm{t}\langle\sec \theta\rangle N_{0} \lambda_{\mathrm{H}} / \lambda_{\text {air }}\right) \times 10^{4} / 8 \pi$, where $\xi_{N}$ $=1.2$ includes the effect of parent particles heavier than protons (Orth and Buffington 1976), $t=11 \mathrm{~g} \mathrm{~cm}^{-2}$ is the atmospheric column thickness, $\langle\sec \theta\rangle=1.08$ corrects for the average zenith angle, $N_{0}$ is Avogadro's number, and the $\lambda$ 's are nuclear collision lengths for hydrogen and air. The final factor converts to $\mathrm{m}^{2} \mathrm{sr}$ and includes a factor of $\frac{1}{2}$ because antineutrons do not decay quickly enough into antiprotons and are themselves unobservable, and hence should not be counted here. Figure 9 shows the scaled result together with another curve which takes account of the Fermi motion of the nucleons in the air nuclei. Gaisser and Levy (1974) discuss this 
effect in the context of $\bar{P}$ production in interstellar space; its inclusion is unimportant except at low energies. The dropping production rate at high energy is due to the power law spectrum of the parent particles. The suppression of low-energy antiprotons results from the kinematics of the production process. Antiprotons cannot be created with an energy close to either beam or target particle since the creation of an extra nucleonantinucleon pair diminishes the available center-of-mass kinetic energy. The dashed curve in Figure 9 was calculated by choosing a random direction and a Gaussian distribution with $\sigma=400 \mathrm{MeV} / c$ of Fermi momentum to define a "target at rest" frame of reference for the solid curve and then converting back to the laboratory frame with a Lorentz transformation. The region of this experiment's sensitivity is indicated by the shaded region; the calculated number of atmospheric antiprotons for this experiment is found to be about $10^{-1}$, a negligible amount.

A cross-check of this calculation is provided by the antiproton events obtained during the final $4 \frac{1}{2}$ hours of the flight, when there was about $18 \mathrm{~g} \mathrm{~cm}^{-2}$ of residual atmosphere instead of 9 . Of the 17 antiproton events, four came during this interval, consistent with the antiprotons having a cosmic origin. This check also is consistent with the supposition that the geomagnetic cutoff was below the effective energy window for this experiment, since the average westward drift of the balloon during the exposure would otherwise have cut

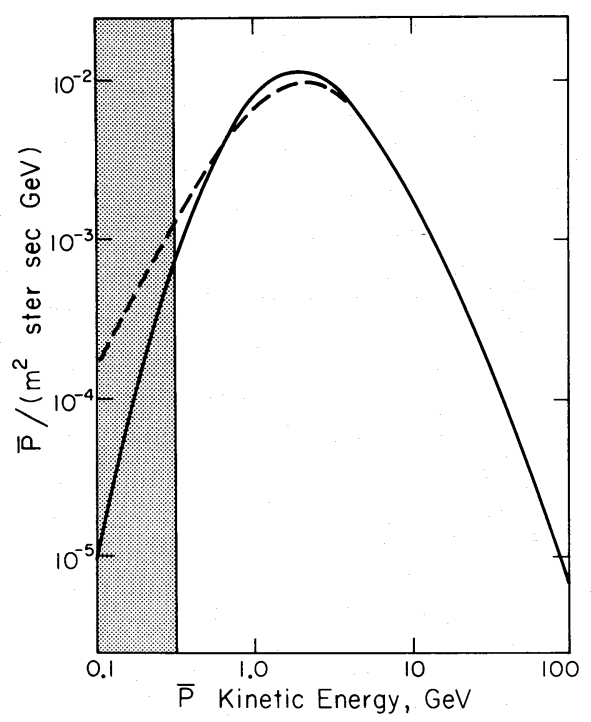

FIG. 9. - Calculation of the expected rate of antiproton production in $11 \mathrm{~g} \mathrm{~cm}^{-2}$ of residual atmosphere. The solid curve is from Stephens (1980), scaled to the case of atmospheric production and converted to the indicated units. The dashed curve is the result of including Fermi motion for the target air nucleons. The shaded region marks the experiment's energy window. off most of the cosmic events before the last few hours of data taking.

\section{d) Comparison with Other Measurements}

The antiproton flux is $1.7 \pm 0.5 \times 10^{-4} \mathrm{~m}^{-2} \mathrm{sr}^{-1} \mathrm{~s}^{-1}$ $\mathrm{MeV}^{-1}$ when corrected to the top of the atmosphere. Previous authors quote a $\bar{P} / P$ ratio rather than a flux. Using the average proton flux of $0.8 \mathrm{~m}^{-2} \mathrm{sr}^{-1} \mathrm{~s}^{-1}$ $\mathrm{MeV}^{-1}$ from Figure 4, this experiment measures a $\bar{P} / P$ ratio of $2.2 \pm 0.6 \times 10^{-4}$, where no extra error is included for uncertainty in the proton flux. Golden et al. (1979) report a $\bar{P} / P$ ratio of $5.2 \pm 1.5 \times 10^{-4}$, Bogomolov et al. (1979) report a ratio of $6 \pm 4 \times 10^{-4}$, and from Apparao (1968) we deduce a $1 \sigma$ upper limit of $5 \times 10^{-4}$ (no events in $\sim 3000$ ). These experiments measure antiprotons between 4.7 and $11.6 \mathrm{GeV}, 2$ and 5 $\mathrm{GeV}$, and 100 and $150 \mathrm{MeV}$, respectively. All of these results are consistent (within a factor of 2) with an antiproton spectrum proportional to the proton spectrum between approximately $100 \mathrm{MeV}$ and $10 \mathrm{GeV}$. The $\bar{P} / P$ ratio measured here could be up to twice as large as reported above, if the assumed proton flux in the denominator was too large, as discussed in $\S \mathrm{Va}$.

\section{e) Solar Modulation}

Urch and Gleeson (1973) have calculated the energy loss of particles reaching $1 \mathrm{AU}$ due to the interaction with the outgoing solar wind. Interpolating their results, we estimate that the antiprotons detected in this experiment typically lost $600 \mathrm{MeV}$ of kinetic energy due to adiabatic deceleration, assuming propagation conditions were similar in 1980 June to the 1969 calculation. The bulk of the antiprotons measured here should have originally had energies between approximately 500 and $1300 \mathrm{MeV}$ in interstellar space, according to these authors. In this model, a $\bar{P} / P$ ratio measured at $1 \mathrm{AU}$ is simply moved up in energy to this higher value. A differential flux measurement is similarly displaced but must also be raised to correct for the phase space change in the differential denominator. More recently, Jokipii and Kopriva (1979) introduced a model of solar modulation which incorporates streaming of the cosmic rays along a current sheet. Although these authors do not disagree with Urch and Gleeson (1973) about the average amount of adiabatic deceleration, they predict a change in the $\bar{P} / P$ ratio from one solar cycle to the next, at the energy of this experiment, of as much as a factor of 4 . The present solar cycle enhances this ratio. We regard the present uncertainty with respect to solar modulation as an impediment to the interpretation of the $\bar{P}$ data presented here, although the major features of the interpretation are not affected significantly by this uncertainty. In the next section, where we discuss this interpretation, we will simply present the measured data points as though solar modulation were not present. 


\section{DISCUSSION}

Figures 10 and 11 show the results of this experiment together with the other measurements, and with calculations of expected antiproton fluxes assuming the standard leaky box model for propagation in the Galaxy. Many of the calculations (Gaisser and Maurer 1973; Badhwar et al. 1975; Szabelski, Wdowczyk, and Wolfendale 1980) present results only in terms of the $\bar{P} / P$ ratio, while Gaisser and Levy (1974) and Stephens (1980) present the absolute flux as well. The difference between the latter calculations could be eliminated by including, in Gaisser and Levy's (1974) calculation, the factor $\xi_{N}=1.34$ (Orth and Buffington 1976) for effects of antiprotons generated by cosmic rays heavier than protons and for effects of helium in the interstellar medium.

Generally, one can see for the standard leaky box model that the measured antiproton fluxes are all larger than expected, dramatically so in the case of the result being reported here. When the solar modulation is taken

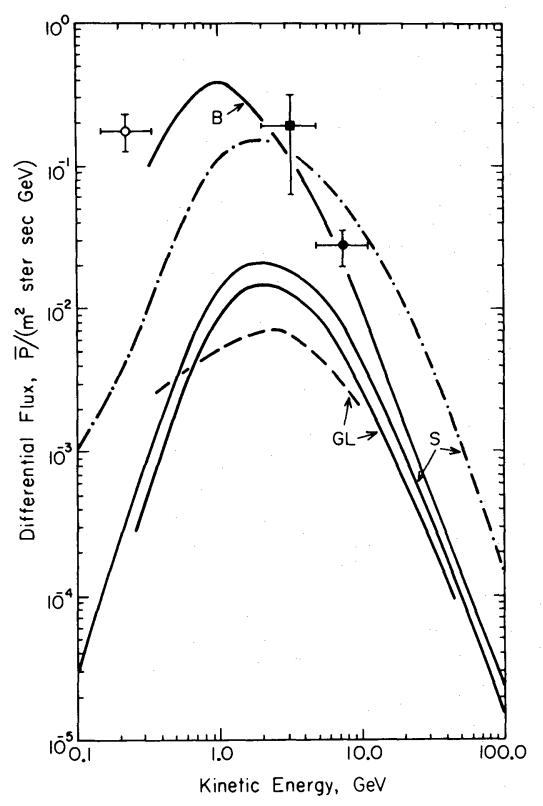

FIG. 10.-A plot of measured and calculated antiproton differential fluxes as a function of energy. The data points shown are: closed circle, Golden et al. (1979); square, Bogomolov et al. (1979); open circle, this experiment. The curve labeled " $\mathrm{B}$ " is from Badhwar et al. (1975), multiplied by $19,000 E_{\text {tot }}^{-2.75}$ to remove the proton flux denominator from the $\bar{P} / P$ ratio. The experimentally measured fluxes have been similarly derived from reported $\bar{P} / P$ ratios. The curves labeled "S" are from Stephens (1980); the lower one is for the standard leaky box model, and the upper one for a closed galaxy model. The curves labeled "GL" are from Gaisser and Levy (1974); the upper one is for the standard leaky box model, and the lower one includes solar modulation. Solar modulation moves antiprotons to lower energy at the apparatus, partially filling in the kinematically suppressed low-energy antiproton flux.

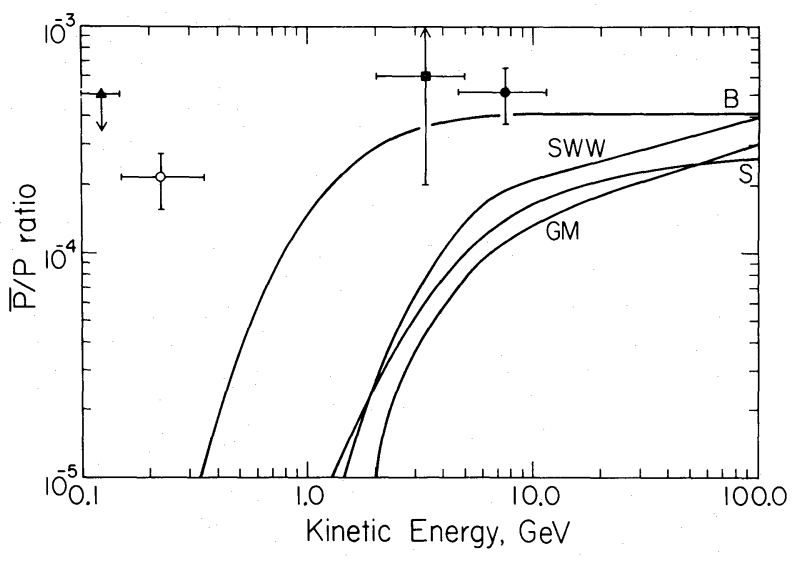

Fig. 11.-A plot of measured and calculated antiproton/proton ratios as a function of energy. Closed circle, Golden et al. (1979); square, Bogomolov et al. (1979); triangle, Apparao (1968); open circle, this experiment. The calculated curves are from " $\mathrm{B}$ " (Badhwar et al. 1975); "SWW" (Szabelski, Wdowczyk, and Wolfendale 1980); "GM" (Gaisser and Maurer 1973); "S" (Stephens 1980). Correction for solar modulation is expected to move the data points to the right by typically $0.6 \mathrm{GeV}$.

into account, correction of the data points in Figure 10 is uncertain, as discussed previously. In Figure 11, this experiment's data point moves to the right by about 600 $\mathrm{MeV}$, which brings it reasonably close to the calculation of Badhwar et al. (1975). However, Stephens (1980, 1981) discusses his reservations about this calculation and has demonstrated that the discrepancy between his result and that of Badhwar et al. (1975) probably originates in different parameterizations of the interaction dynamics. Badhwar et al. (1975) use parameters which tend to overestimate the production at low energy. This accounts for their prediction of a much larger antiproton flux near $1 \mathrm{GeV}$. When the effects of solar modulation are taken into account, the experimental measurements are typically only a factor of 2 above the predictions of Badhwar et al. (1975), but we feel that their calculation is not correct at low energy and that the other curves represent the best present day prediction. About a quarter of the interactions giving rise to antiprotons in interstellar space take place with helium nuclei as the target, and the same Fermi motion included previously with Stephens's production spectrum to calculate atmospheric antiprotons is present in these helium nuclei. However, it is clear from Figure 9 that this minor detail is not of much help in closing the gap between experiment and theory. Indeed, the integrated flux of Stephens's leaky box curve is only $0.15 \bar{P} \mathrm{~m}^{-2}$ $\mathrm{sr}^{-1} \mathrm{~s}^{-1}$, and the flux reported here is a quarter of that, within only a small energy interval. When all measurements are included, typically 10 times Stephens's calculated integral flux is required simply to provide sufficient numbers of antiprotons. Stephens $(1980,1981)$ has suggested that a combination of the leaky box 
model with the closed galaxy model of Rasmussen and Peters (1975; Peters and Westergaard 1977) could give a fit to the data of Golden et al. (1979) and Bogomolov et al. (1979). We have included on Figure 10 his calculation for this model. Essentially, the closed galaxy model increases the expected number of antiprotons by introducing a tenfold increase in effective path length traversed by the parent protons. In the closed galaxy model, antiprotons continue to build up until their production equals their destruction through annihilation. Although this would provide an adequate integral $\bar{P}$ flux, the increase in expected antiproton flux still fails to explain the number observed at low energy. The combined antiproton measurements show little sign of the expected low-energy kinematic suppression.

Gaisser and Levy (1974) speculate that primary antiprotons should not show the kinematic suppression and instead should have a power law similar to that observed for protons. It is, of course, possible that primary antiprotons are the explanation for the measurement reported here, but this is difficult to reconcile with the absence of antihelium. Regions of bulk antimatter, as in the models reviewed by Steigman (1976), ought to synthesize and accelerate antihelium and heavier antinuclei in the same proportions found in the common matter cosmic rays. If the $\overline{\mathrm{He}} / \bar{P}$ ratio were the same as the $\mathrm{He} / P$ ratio, then we should have observed even more $\overline{\mathrm{He}}$ events in this experiment than we did $\bar{P}$ events because of the larger geometry factor for $\overline{\mathrm{He}}$. Instead, we observed none, thus putting an upper limit (Fig. 8) on $\overline{\mathrm{He}} / \mathrm{He}$, which agrees with higher energy and heavier nucleus results. The $95 \%$ confidence $(\overline{\mathrm{He}} / \mathrm{He}) /(\bar{P} / P)$ ratio upper limit is 0.1 . This restricts a primary explanation for the antiprotons reported here, although the relative rarity of heavier antinuclei could also be explained if the cosmic-ray antimatter had to traverse a thick slab of material $\left(\gtrsim 20 \mathrm{~g} \mathrm{~cm}^{-2}\right)$ in reaching us. Another possibility is the creation of antiprotons, but not heavier antinuclei, directly by some exotic process in some cosmic-ray sources.

Szabelski, Wdowczyk, and Wolfendale (1980) have discussed a possible alternative explanation for the result being presented here. If there were stochastic, energy-changing interactions of cosmic rays while propagating in the Galaxy, these many energy changes might wash out the kinematically suppressed portion of the low-energy antiproton spectrum and could give rise to a power law spectrum similar to that of the more plentiful cosmic rays. In the past there have been several mechanisms suggested which could have this effect (Fermi 1949; Ginzburg and Syrovatskii 1964). The featureless spectra of most other cosmic-ray species do not allow the separation of interstellar propagation effects from an original injection spectrum, since the latter is unknown. However, the spectrum of secondary antiprotons is calculable, and in the absence of spectrum-altering processes it should appear as shown in the lower curves of Figures 10 and 11. The poor match in spectral shape between the data and theory suggest that stochastic, energy-changing processes may be an important part of cosmic-ray propagation in the Galaxy, at least at energies up to several GeV. Several other species of secondary cosmic rays $\left({ }^{49} \mathrm{~V}\right.$ and deuterons) should also have unusual spectral features as a result of production cross section variations (Raisbeck et al. 1973; Meyer 1974). These features may also be altered if this hypothesis is correct. However, if the closed galaxy model is employed to explain the large total $\bar{P}$ flux and if the heavier cosmic rays are mostly due to the "young" component, these daughters may not be old enough for the stochastic processes to have altered their spectral features.

This work was supported by grants NGR 05-003-553 and NGR 05-002-160 from the National Aeronautics and Space Administration and by the University of California's Lawrence Berkeley Laboratory, supported by the Department of Energy. The balloon flight operation was conducted by members of the National Scientific Balloon Facility in Palestine, Texas. Project authority for the Canadian balloon flight, together with liaison support, was provided by the National Research Council of Canada, Space Research Facilities Branch. Use of the mountaintop laboratory was possible through the hospitality of New Mexico State University at Las Cruces. Logistical and technical support was provided during the mountaintop calibration by members of Sacramento Peak Observatory. We would like especially to acknowledge the devoted labor of John Yamada, John Gibson, and Hal Dougherty of the Space Sciences Laboratory at Berkeley who carried out much of the construction, test, and field operation support for the experiment. The importance of deuterons and tritons as a potential trigger background was first pointed out to us by Ray Hagstrom. George F. Smoot, Phil M. Lubin, and Charles D. Orth all made important contributions to the early design of the apparatus. We thank L. W. Alvarez, R. W. Birge, A. G. Opp, and P. B. Price for much support and encouragement. Finally, we are pleased to thank R. A. Mewaldt, T. A. Prince, E. C. Stone, R. E. Vogt, and other members of the Space Radiation Laboratory at Caltech for careful reading of the manuscript and much advice and criticism received during the data analysis phase of this experiment.

\section{REFERENCES}

Agnew, L. E. et al. 1960, Phys. Rev., 118, 1371.

Apparao, M. V. K. 1968, Proc. 10th International Cosmic Ray Conf., Calgary; Canadian J. Phys., 46, S654.

Badhwar, G. D., Golden, R. L., Brown, M. L., and Lacy, J. L. 1975, Ap. Space Sci., 37, 283. 
Badhwar, G. D., Golden, R. L., Lacy, J. L., Zipse, J. E., Daniel, R. R., and Stephens, S. A. 1978, Nature, 274, 137.

Barber, H. B. 1976, Ph.D. thesis, University of Arizona.

Bogomolov, E. A., Lubyanaya, N. D., Romanov, V. A., Stepanov, S. V., and Shulakova, M. S. 1979, Proc. 16th International Cosmic Ray Conference, Kyoto, 1, 330.

Buffington, A., Orth, C. D., and Smoot, G. F. 1975, Ap. J., 199, 669.

Buffington, A., Pennypacker, C. R., Lubin, P. M., and Smoot, G. F. 1979, Proc. 16th International Cosmic Ray Conference, Kyoto, 1, 337.

Ekspong, A. G., Frisk, Å., Nilsson, S., and Ronne, B. E. 1961, Nucl. Phys., 22, 353.

Fanselow, J. L., Hartman, R. C., Hildebrand, R. H., and Meyer, Peter. 1969, Ap. J., 158, 771.

Fanselow, J. L., and Stone, E. C. 1972, J. Geophys. Res., 77, 3999.

Fermi, E. 1949, Phys. Rev., 75, 1169.

Gaisser, T. K., and Levy, E. H. 1974, Phys. Rev., D, 10, 1731

Gaisser, T. K., and Maurer, R. H. 1973, Phys. Rev. Letters, 30, 1264.

Ginzburg, V. L., and Syrovatskii, S. I. 1964, The Origin of Cosmic Rays (New York: Pergamon Press).

Golden, R. L., Horan, S., Mauger, B. G., Badhwar, G. D., Lacy, J. L., Stephans, S. A., Daniel, R. R., and Zipse, J. E. 1979, Phys. Rev. Letters, 43, 1196.

Janni, J. F. 1966, AFWL-TR-65-150, Air Force Weapons Laboratory, Kirtland AFB, New Mexico.

Jokipii, J. R., and Kopriva, D. A. 1979, Ap. J., 234, 384

Metropolis, N., Bivins, R., Storm, M., Turkevich, A., Miller, J. M., and Friedlander, G. 1958, Phys. Rev., 110, 185.

Meyer, J. P. 1974, Ph.D. thesis, Paris University, Orsay.
Ormes, J. F., and Webber, W. R. 1968, J. Geophys. Res., 73, 4231. Orth, C. D., and Buffington, A. 1976, Ap. J., 206, 312.

Pennypacker, C. R., Buffington, A., Lubin, P. M., and Smoot, G. F. 1979, Proc. 16th International Cosmic Ray Conference, Kyoto, 12, 61 .

Peters, B., and Westergaard, N. J. 1977, Ap. Space Sci., 48, 21

Powell, C. F., Fowler, P. H., and Perkins, D. H. 1959, The Study of Elementary Particles by the Photographic Method (New York: Pergamon Press).

Raisbeck, G., Perron, C., Toussaint, J., and Yiou, F. 1973, Proc. 13th International Cosmic Ray Conference, Denver, 1, 534.

Rasmussen, I. L., and Peters, B. 1975, Nature, 258, 412.

Renner, B. 1966, M.N.R.A.S., 133, 197.

Rygg, T. A., and Earl, J. A. 1971, J. Geophys. Res., 76, 7445.

Shea, M. A., Smart, D. F., and Carmichael, H. 1976, AFGL-TR76-0115, Hanscom AFB, Massachusetts.

Smoot, G. F., Buffington, A., and Orth, C. D. 1975, Phys. Rev. Letters, 35, 258

Steigman, G. A. 1976, Ann. Rev. Astr. Ap., 14, 339.

Stephens, S. A. 1980, preprint SP/IR-80-2, Tata Institute; submitted to Ap. Space Sci. 1981, Nature, 289, 267.

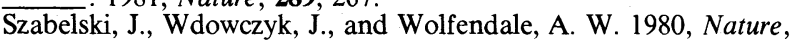
285, 386.

Trower, W. P. 1966, High Energy Particle Data, Vol. 4 (UCRL2426 .

Urch, I. H., and Gleeson, L. J. 1972, Ap. Space Sci., 17, 426 1973, Ap. Space Sci., 20, 177.

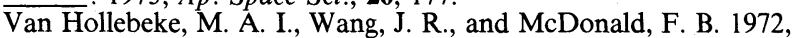
J. Geophys. Res., 77, 6881

ANDrew Buffington and StePhen M. Schindler: California Institute of Technology, Mail Code 220-47, Pasadena, CA 91125

CARLton R. PennYPACKer: Room 50-232, Lawrence Berkeley Laboratory, Berkeley, CA 94720 